\title{
Journal of Hematology \& Oncology reviewer acknowledgement 2013
}

Delong Liu

\section{Contributing reviewers}

The editors of Journal of Hematology \& Oncology would like to thank all our reviewers who have contributed to the journal in Volume 6 (2013).

Seema Agarwal

United States of America

Akintunde Akinleye

United States of America

Donatella Aldinucci

Italy

Robert Amato

United States of America

Ningfei An

United States of America

Peter Anderson

United States of America

Rami Aqeilan

Israel

Philippe Arnaud

France

Francis Ayuk

Germany

Jeff Barrett

United States of America

Surinder Batra

United States of America

Paul Beaudry

Canada
Leonidas Benetatos
Greece

Paolo Bernasconi

Kazakhstan

Zwi Berneman

Belgium

Jaap Jan Boelens

Netherlands

Attilio Bondanza

Italy

Elisa Boscolo

United States of America

Joseph Brandwein

Canada

Adam Brufsky

United States of America

Brad Bryan

United States of America

Nicholas Burgis

United States of America

Thomas Burmeister

Germany

Alan Burnett

United Kingdom
Amanda Cashen

United States of America

Richard Cathomas

Switzerland

Ling Cen

United States of America

Hong Chang

Canada

Nan-Shan Chang

Taiwan

Changyan Chen

United States of America

\section{Liguang Chen}

United States of America

\section{Liang Cheng}

United States of America

Alexander Chi

United States of America

\section{Andy Chien}

United States of America

Dai Chihara

Japan

William Cho

Hong Kong

Correspondence: editor@jhoonline.org

New York Medical College, New York, USA 
Michael Chopp

United States of America

Magdalena Chrzanowska-Wodnicka

United States of America

Shih-Sung Chuang

Taiwan

Fabrice Cognasse

France

Gaetano Corazzelli

Italy

Mei-Zhen Cui

United States of America

Nico Dantuma

Sweden

Evandro De Azambuja

Belgium

Christophe De Romeuf

France

Adriana De Siervi

Argentina

Paolo Dion

Italy

Angela Dispenzieri

United States of America

Jixin Dong

United States of America

Caigan Du

Canada

James Eddy

United States of America

Stephan Ensminger

Germany

Mehmet Ali Erkurt

Turkey

Beth Faiman

United States of America

Marco Falasca

United Kingdom

Isabella Faraoni

Italy

Ru Feng

China

Michael Flister

United States of America
Alessandro Fornari

Italy

Nathan Fowler

United States of America

Massimo Franchini

Italy

Arnold Freedman

United States of America

Christopher French

United States of America

Stefan Fröhling

Germany

Teruhiko Fujii

Japan

Yutaka Fujiwara

Japan

Seiji Fukuda

Japan

Shirish Gadgeel

United States of America

Gianluca Gaidano

Italy

Peter Gaines

United States of America

Kristen Ganjoo

United States of America

Christian Geisler

Denmark

Jean-Pierre Gerard

France

Laurence Gerard

France

Herve Ghesquieres

France

Stephane Giraudier

France

Stefania Gobessi

Italy

Abhijit Godbole

United States of America

Josee Golay

Italy

Steven Gore

United States of America
Andre Goy

United States of America

Richard Greil

Austria

Baiwei Gu

United States of America

Göhring Gudrun

Germany

Angelo Guerrasio

Italy

Thomas Habermann

United States of America

Michiyuki Hakozaki

Japan

Maria Francisca Ham

Indonesia

Tanja Hartmann

Austria

Robert Hasserjian

United States of America

Aiwu He

United States of America

Charles Hemenway

United States of America

Vera Hirsh

Canada

Georg Hopfinger

Austria

Chung-Tsen Hsueh

United States of America

Jiong $\mathrm{Hu}$

China

He Huang

China

Liang Huang

United States of America

Yujie Huang

United States of America

Sara Hurvitz

United States of America

Shinsuke Iida

Japan

Martino Introna

Italy 


\begin{tabular}{|c|c|c|}
\hline $\begin{array}{l}\text { Fumihiro Ishida } \\
\text { Japan }\end{array}$ & $\begin{array}{l}\text { Andrei Kramerov } \\
\text { United States of America }\end{array}$ & $\begin{array}{l}\text { Irene Lorand-Metze } \\
\text { Brazil }\end{array}$ \\
\hline $\begin{array}{l}\text { Yasuo Iwadate } \\
\text { Japan }\end{array}$ & $\begin{array}{l}\text { Eric Kraut } \\
\text { United States of America }\end{array}$ & $\begin{array}{l}\text { Nancy Louis } \\
\text { United States of America }\end{array}$ \\
\hline $\begin{array}{l}\text { Jun Iwashita } \\
\text { Japan }\end{array}$ & $\begin{array}{l}\text { Geoffrey Ku } \\
\text { United States of America }\end{array}$ & $\begin{array}{l}\text { Cynthia Ma } \\
\text { United States of America }\end{array}$ \\
\hline $\begin{array}{l}\text { Elias Jabbour } \\
\text { United States of America }\end{array}$ & $\begin{array}{l}\text { Karin Kurnik } \\
\text { Germany }\end{array}$ & $\begin{array}{l}\text { Daoxin Ma } \\
\text { China }\end{array}$ \\
\hline $\begin{array}{l}\text { Tang-Her Jaing } \\
\text { Taiwan }\end{array}$ & $\begin{array}{l}\text { Junya Kuroda } \\
\text { Japan }\end{array}$ & $\begin{array}{l}\text { Xiaotu Ma } \\
\text { United States of America }\end{array}$ \\
\hline $\begin{array}{l}\text { Gaetan Jego } \\
\text { France }\end{array}$ & $\begin{array}{l}\text { Mateusz Kurzawski } \\
\text { Poland }\end{array}$ & $\begin{array}{l}\text { Anne-Marie Madec } \\
\text { France }\end{array}$ \\
\hline $\begin{array}{l}\text { Yuxia Jia } \\
\text { United States of America }\end{array}$ & $\begin{array}{l}\text { Toshihiro Kushibiki } \\
\text { Japan }\end{array}$ & $\begin{array}{l}\text { Michael Makris } \\
\text { United Kingdom }\end{array}$ \\
\hline $\begin{array}{l}\text { Enzi Jiang } \\
\text { United States of America }\end{array}$ & $\begin{array}{l}\text { Intidhar Labidi-Galy } \\
\text { United States of America }\end{array}$ & $\begin{array}{l}\text { Elisabet Esteve Manasanch } \\
\text { United States of America }\end{array}$ \\
\hline $\begin{array}{l}\text { Yongping Jiang } \\
\text { China }\end{array}$ & $\begin{array}{l}\text { Gurpreet Lamba } \\
\text { United States of America }\end{array}$ & $\begin{array}{l}\text { Ferdinando Mannello } \\
\text { Italy }\end{array}$ \\
\hline $\begin{array}{l}\text { Jie Jin } \\
\text { China }\end{array}$ & $\begin{array}{l}\text { Manuel Lemos } \\
\text { Portugal }\end{array}$ & $\begin{array}{l}\text { Pier Mannuccio Mannucci } \\
\text { Italy }\end{array}$ \\
\hline $\begin{array}{l}\text { Liang Jin } \\
\text { Australia }\end{array}$ & $\begin{array}{l}\text { Ji-Liang Li } \\
\text { United Kingdom }\end{array}$ & $\begin{array}{l}\text { Tibiletti Maria Grazia } \\
\text { Italy }\end{array}$ \\
\hline $\begin{array}{l}\text { Qihuang Jin } \\
\text { United States of America }\end{array}$ & $\begin{array}{l}\text { Tianhong Li } \\
\text { China }\end{array}$ & $\begin{array}{l}\text { Ana Marques } \\
\text { United Kingdom }\end{array}$ \\
\hline $\begin{array}{l}\text { Grace Kao } \\
\text { United States of America }\end{array}$ & $\begin{array}{l}\text { Yangqiu Li } \\
\text { China }\end{array}$ & $\begin{array}{l}\text { William Matsui } \\
\text { United States of America }\end{array}$ \\
\hline $\begin{array}{l}\text { Sabine Kasimir-Bauer } \\
\text { Germany }\end{array}$ & $\begin{array}{l}\text { Zhong Li } \\
\text { China }\end{array}$ & $\begin{array}{l}\text { Fabrizio Mattei } \\
\text { Italy }\end{array}$ \\
\hline $\begin{array}{l}\text { Masaru Katoh } \\
\text { Japan }\end{array}$ & $\begin{array}{l}\text { Sharon Liang } \\
\text { United States of America }\end{array}$ & $\begin{array}{l}\text { Sandra Mcallister } \\
\text { United States of America }\end{array}$ \\
\hline $\begin{array}{l}\text { Chul Geun Kim } \\
\text { Korea, South }\end{array}$ & $\begin{array}{l}\text { Evi Lianidou } \\
\text { Greece }\end{array}$ & $\begin{array}{l}\text { Michal Mego } \\
\text { United States of America }\end{array}$ \\
\hline $\begin{array}{l}\text { Won Seog Kim } \\
\text { Korea, South }\end{array}$ & $\begin{array}{l}\text { Wolfgang Link } \\
\text { Portugal }\end{array}$ & $\begin{array}{l}\text { Shu Meng } \\
\text { United States of America }\end{array}$ \\
\hline $\begin{array}{l}\text { Hitoshi Kiyoi } \\
\text { Japan }\end{array}$ & $\begin{array}{l}\text { Eva Lion } \\
\text { Belgium }\end{array}$ & $\begin{array}{l}\text { Adam Metwalli } \\
\text { United States of America }\end{array}$ \\
\hline $\begin{array}{l}\text { Wolfram Klapper } \\
\text { Germany }\end{array}$ & $\begin{array}{l}\text { Delong Liu } \\
\text { United States of America }\end{array}$ & $\begin{array}{l}\text { Stefan Meyer } \\
\text { United Kingdom }\end{array}$ \\
\hline $\begin{array}{l}\text { Young Hyeh Ko } \\
\text { Korea, South }\end{array}$ & $\begin{array}{l}\text { Hong Liu } \\
\text { United States of America }\end{array}$ & $\begin{array}{l}\text { Joseph Mikhael } \\
\text { United States of America }\end{array}$ \\
\hline $\begin{array}{l}\text { Michael Koldehoff } \\
\text { Germany }\end{array}$ & $\begin{array}{l}\text { Jianguo Liu } \\
\text { United States of America }\end{array}$ & $\begin{array}{l}\text { Michele Milella } \\
\text { Italy }\end{array}$ \\
\hline $\begin{array}{l}\text { Hasan Korkaya } \\
\text { United States of America }\end{array}$ & $\begin{array}{l}\text { Cristiana Lo Nigro } \\
\text { Italy }\end{array}$ & $\begin{array}{l}\text { Ken Mills } \\
\text { United Kingdom }\end{array}$ \\
\hline
\end{tabular}

Fumihiro Ishida

Japan

Jun Iwashita

Elias Jabbour

Tang-Her Jaing

Taiwan

Gaetan Jego

Yuxia Jia

Enzi Jiang

United States of America

Yongping Jiang

Jie Jin

Liang Jin

Australia

Qihuang Jin

nited States of America

Grace Kao

Sabine Kasimir-Bauer

Germany

Masaru Katoh

Chul Geun Kim

Won Seog Kim

Korea, South

Hitoshi Kiyoi

Japan

Wolfram Klapper

Young Hyeh Ko

Michael Koldehoff

Germany

United States of America
Andrei Kramerov

Eric Kraut

of America

Karin Kurnik

Junya Kuroda

Japan

Mateusz Kurzawski

Toshihiro Kushibiki
Japan

Intidhar Labidi-Galy

United States of America

Gurpreet Lamba

Manuel Lemos

Ji-Liang Li

United Kingdom

hong $\mathrm{Li}$

Yangqiu Li

Zhong Li

China

Sharon Liang

Evi Lianidou

Portugal

Delong Liu

Hong Liu

United States of America

ianguo Liu

Cristiana Lo Nigro

United Kingdom 


\author{
Mark Minden \\ Canada
}

Hans Minderman

United States of America

Tony Mok

Hong Kong

David Mole

United Kingdom

Hiroyuki Momota

Japan

Tammy Morrish

United States of America

Hasan Mukhtar

United States of America

Steffan Nawrocki

United States of America

John Nelson

United States of America

Seiji Niho

Japan

Shozo Nishida

Japan

Bodil Norrild

Denmark

Rajesh Kumar NV

United States of America

Massimo Offidani

Italy

Seiichi Okabe

Japan

Yasuhiro Oki

United States of America

Horatiu Olteanu

United States of America

Toru Ouchi

United States of America

Thomas Pabst

Switzerland

Chin-Chen Pan

Taiwan

Helen Papadaki

Greece

Tomas Papajik

Czech Republic
Samir Parekh

United States of America

Upendra Parvathaneni

United States of America

Tejal Patel

United States of America

Swati Pathak

United States of America

Jun Peng

China

Christian Peschel

Germany

Katharina Pfistershammer

Austria

Rocco Piazza

Italy

Hélène A Poirel

Belgium

Guy Pratt

United Kingdom

Vít Procházka

Czech Republic

Soham Puvvada

United States of America

Anne Quillet-Mary

France

Farhang Rabbani

United States of America

Shamudheen Rafiyath

United States of America

Pablo Ramirez

Chile

William Redmond

United States of America

Ruibao Ren

United States of America

Adam Resnick

United States of America

Ryan Riddle

United States of America

Tadeusz Robak

Poland

Jesse Roman

United States of America
Stefano Rosso

Italy

John Ryan

United States of America

Nabil Saba

United States of America

Lorena Sánchez-Martín

Spain

Valeria Santini

Italy

Yorifumi Satou

United Kingdom

Yogen Saunthararajah

United States of America

Kerry Savage

Canada

Nic Savaskan

Germany

Heiner Schäfer

Germany

Christian Scharenberg

Sweden

Mirta Schattner

Argentina

Gary Schiller

United States of America

Bin Shan

United States of America

Mahesh Sharma

United States of America

Anieta Sieuwerts

Netherlands

David Snyder

United States of America

Brigitte Sola

France

Tobias Sperka

Germany

James Spicer

United Kingdom

Daniel Starczynowski

United States of America

Peter Steinberger

Austria 


\section{Cordula Stover \\ United Kingdom \\ K. Stephen Suh \\ United States of America \\ Anders Sundan \\ Norway \\ Ritsuro Suzuki \\ Japan}

Arthur Sytkowski

United States of America

Philippe Szankasi

United States of America

Shinichiro Takahashi
Japan

Jin Takeuchi

Japan

Dipti Talaulikar

Australia

Keiji Tanese

Japan

Chih-Hsin Tang

Taiwan

Matthias Tenbusch

Germany

John Thompson

United States of America

Kirsty Thomson

United Kingdom

Kenneth To

Hong Kong

Mariko Tomita

Japan

Naoto Tomita

Japan

Hai Tran

United States of America

Martin Trepel

Germany

Yang Tsai Sheng

Taiwan

William Tse

United States of America

Alessandra Tucci

Italy
Togas Tulandi

Canada

Shinya Uchino

Japan

Henning Ulrich

Brazil

Antonis Valachis

Greece

Ben Valdez

United States of America

Eric Van Den Neste

Belgium

Johan Van Krieken

Netherlands

K. David Voduc

Canada

Maria Teresa Voso

Italy

Domagoj Vucic

United States of America

Christine Walsh

United States of America

Dazhi Wang

United States of America

Donghai Wang

United States of America

Gang Wang

China

Kankan Wang

China

Xin Wang

China

Ya Wang

United States of America

Zilong Wen

Hong Kong

Meir Wetzler

United States of America

Andre Willasch

Germany

Thian-Sze Wong

Hong Kong

Gang Wu

China
Dazhong Xu

United States of America

Qingwen Xu

United States of America

Teng Xu

United States of America

Motoko Yamaguchi

Japan

Ying Yan

United States of America

David Yang

United States of America

Yibin Yang

United States of America

Jean Yared

United States of America

M. James You

United States of America

Ken Young

United States of America

Herbert Yu

United States of America

Bo Yuan

Japan

Zihua Zeng

United States of America

Fenghuang Zhan

United States of America

Chengcheng Zhang

United States of America

Dongwei Zhang

United States of America

Hua Zhang

China

Jiangwei Zhang

United States of America

Jianke Zhang

United States of America

Xiaolei Zhang

United States of America

Xiaowei Zhang

United States of America

Yi Zhang

United States of America 
Yuxia Zhang

United States of America

Qian Zhao

China

Zhizhuang Joe Zhao

United States of America
Xiaoyong Zheng

United States of America

Daohong Zhou

United States of America
Zhengping Zhuang

United States of America

Mario Zoratti

Italy doi:10.1186/1756-8722-7-12

Cite this article as: Liu: Journal of

Hematology \& Oncology reviewer

acknowledgement 2013. Journal of

Hematology \& Oncology 2014 7:12. 